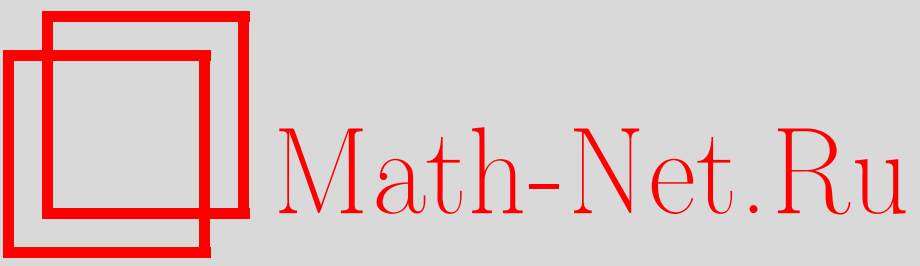

Д. В. Берзин, Инварианты коприсоединенного представления для алгебр Ли некоторого специального вида, УМН, 1996, том 51, выпуск 1, 141-142

DOI: https://doi.org/10.4213/rm923

Использование Общероссийского математического портала Math-Net.Ru подразумевает, что вы прочитали и согласны с пользовательским соглашением

http://www.mathnet.ru/rus/agreement

Параметры загрузки:

IP: 54.237 .206 .68

26 апреля 2023 г., 16:07:23 


\title{
ИНВАРИАНТЫ КОПРИСОЕДИНЕННОГО ПРЕДСТАВЛЕНИЯ ДЛЯ АЛГЕБР ЛИ НЕКОТОРОГО СПЕЦИАЛЬНОГО ВИДА
}

\author{
Д. В. БЕРЗИн
}

Введение. Пусть $\Theta$ - алгебра Ли, $\operatorname{dim} \Theta=n(n \geqslant 2), \Theta^{*}-$ пространство, сопряженное с $\Theta, G$ - группа Ли, ассоциированная с алгеброй Ли $\Theta$.

Пусть $C_{i j}^{k}$ - структурньй тензор алгебры Ли $\Theta$ в базисе $e_{i}, x_{i}$ - координаты в $\Theta^{*}$, соответствующие базису $e^{1}, \ldots, e^{n}$ пространства $\Theta^{*}$, такому, что $e^{i}\left(e_{j}\right)=\delta_{j}^{i}$.

ОпредЕлЕниЕ. Аналитическая функция $F$ на $\Theta^{*}$ называется инвариантом коприсоединенного представления группы $G$, если $F(f)=F\left(\operatorname{Ad}_{g}^{*} f\right)$ для любых $g \in G, f \in \Theta^{*}$.

ПредЛОжение (см. [1, гл. 5]). Аналитическая функиия $F$ на $\Theta^{*}$ является инвариантом коприсоединенного представления группь Ли $G$, ассочиированной $c \Theta$, тогда и только тогда, когда

$$
\left\{\begin{array}{l}
C_{1 j}^{k} x_{k} \frac{\partial F}{\partial x_{j}}=0 \\
\cdots \cdots \\
C_{n j}^{k} x_{k} \frac{\partial \dot{F}}{\partial x_{j}}=0
\end{array}\right.
$$

Настоящая работа посвящена нахождению инвариантов коприсоединенного представления в случае, когда алгебра Ли определяется билинейной и кососимметрической формой, задаваемой соотношениями:

$$
\left(C_{n j}^{k}\right)=\left(\begin{array}{cccccc}
\lambda & 1 & & & & 0 \\
& \lambda & 1 & & & \\
& & \lambda & & & \\
& & & \ddots & & \\
& & & & \lambda & 1 \\
0 & & & & & \lambda
\end{array}\right), \quad \begin{aligned}
& k, j=1, \ldots, n-1, \\
& C_{i j}^{k}=0 \text { при } i \neq n, \quad j \neq n .
\end{aligned}
$$

1. Случай нулевых собственных значений. Рассмотрим сначала случай $\lambda=0$. Система (1) примет вид системы из $(n-1)$ уравнения, из первых $(n-2)$ уравнений которой следует, что инварианты не зависят от $x_{n}$.

Последнее уравнение этой системы имеет вид:

$$
x_{1} \frac{\partial F}{\partial x_{2}}+x_{2} \frac{\partial F}{\partial x_{3}}+\cdots+x_{n-2} \frac{\partial F}{\partial x_{n-1}}=0 .
$$

Это уравнение эквивалентно системе обыкновенных дифференциальных уравнений

$$
\frac{d x_{2}}{x_{1}}=\frac{d x_{3}}{x_{2}}=\cdots=\frac{d x_{n-1}}{x_{n-2}}=\frac{d s}{s}
$$

в том смысле, что любой первый интеграл $C$ системы (3) есть решение $F$ системы $(2)$, и наоборот.

$F_{1}=C_{1}=x_{1}$ - этот инвариант находится сразу. Сравнивая первый и второй, затем первый и третий члены в (3), получаем:

$$
F_{2}=C_{2}=\frac{x_{2}^{2}}{2}-x_{1} x_{3}, \quad F_{3}=C_{3}=-\frac{x_{2}^{3}}{3}+x_{1} x_{2} x_{3}-x_{1}^{2} x_{4}
$$

Опуская длинные выкладки, выпишем в явном виде инварианты для алгебр Ли вплоть до размерности $n=11$ :

$$
\begin{aligned}
& F_{4}=\frac{x_{2}^{4}}{8}-\frac{x_{1} x_{2}^{2} x_{3}}{2}+x_{1}^{2} x_{2} x_{4}-x_{1}^{3} x_{5}, \\
& F_{5}=-\frac{x_{2}^{5}}{30}+\frac{x_{1} x_{2}^{3} x_{3}}{6}-\frac{x_{1}^{2} x_{2}^{2} x_{4}}{2}+x_{1}^{3} x_{2} x_{5}-x_{1}^{4} x_{6},
\end{aligned}
$$




$$
\begin{aligned}
F_{6}= & \frac{x_{2}^{6}}{144}-\frac{x_{1} x_{2}^{4} x_{3}}{24}+\frac{x_{1}^{2} x_{2}^{3} x_{4}}{6}-\frac{x_{1}^{3} x_{2}^{2} x_{5}}{2} \\
& +x_{1}^{4} x_{2} x_{6}-x_{1}^{5} x_{7}, \\
F_{7}= & -\frac{x_{2}^{7}}{840}+\frac{x_{1} x_{2}^{5} x_{3}}{5 !}-\frac{x_{1}^{2} x_{2}^{4} x_{4}}{4 !}+\frac{x_{1}^{3} x_{2}^{3} x_{3}}{3 !}-\frac{x_{1}^{4} x_{2}^{2} x_{6}}{2} \\
& +x_{1}^{5} x_{2} x_{7}-x_{1}^{6} x_{8}, \\
F_{8}= & \frac{x_{2}^{8}}{5760}-\frac{x_{1} x_{2}^{6} x_{3}}{6 !}+\frac{x_{1}^{2} x_{2}^{5} x_{4}}{5 !}-\frac{x_{1}^{3} x_{2}^{4} x_{5}}{4 !} \\
& +\frac{x_{1}^{4} x_{2}^{3} x_{6}}{3 !}-\frac{x_{1}^{5} x_{2}^{2} x_{7}}{2}+x_{1}^{6} x_{2} x_{8}-x_{1}^{7} x_{9}, \\
F_{9}= & -\frac{x_{2}^{9}}{45360}+\frac{x_{1} x_{2}^{7} x_{3}}{7 !}-\frac{x_{1}^{2} x_{2}^{6} x_{3}}{6 !}+\frac{x_{1}^{3} x_{2}^{5} x_{5}}{5 !}-\frac{x_{1}^{4} x_{2}^{4} x_{6}}{4 !} \\
& +\frac{x_{1}^{5} x_{2}^{3} x_{7}}{6}-\frac{x_{1}^{6} x_{2}^{2} x_{8}}{2}+x_{1}^{7} x_{2} x_{9}-x_{1}^{8} x_{10} .
\end{aligned}
$$

TeOpema 1.

(4) $F_{r}=(-1)^{r} \frac{x_{2}^{r}}{r((r-2) !)}+\sum_{k=1}^{r-2}(-1)^{r-k} \frac{x_{1}^{k} x_{2}^{r-k-1} x_{k+2}}{(r-k-1) !}-x_{1}^{r-1} x_{r+1}, \quad r=4,5, \ldots, n-2$.

2. Случай, когда $\lambda$ отличны от нуля. Разберем теперь случай $\lambda \neq 0$. Система (1) в этом случае примет вид системы $n$ уравнений, из первых $(n-1)$ уравнений которой следует, что инварианты не зависят от $x_{n}$. Последнее уравнение этой системы имеет вид:

$$
\lambda x_{1} \frac{\partial F}{\partial x_{1}}+\left(x_{1}+\lambda x_{2}\right) \frac{\partial F}{\partial x_{2}}+\cdots+\left(x_{n-2}+\lambda x_{n-1}\right) \frac{\partial F}{\partial x_{n-1}}=0 .
$$

Оно эквивалентно (в указанном выше смысле) системе обькновенных дифференциальных уравнений:

$$
\frac{d x_{1}}{\lambda x_{1}}=\frac{d x_{2}}{x_{1}+\lambda x_{2}}=\cdots=\frac{d x_{n-1}}{x_{n-2}+\lambda x_{n-1}}=\frac{d s}{s} .
$$

Сравнивая первый и второй, первый и третий, затем первый и четвертый члены в (6), получаем: $F_{1}=C_{1}=x_{1} \exp \left(-\lambda x_{2} / x_{1}\right), F_{2}=C_{2}=\frac{x_{2}^{2}}{2 x_{1}}-\frac{x_{3}}{x_{1}}, F_{3}=\frac{1}{x_{1}^{3}}\left(-\frac{1}{3} x_{2}^{3}+x_{1} x_{2} x_{3}-x_{1}^{2} x_{4}\right)$.

TEOPEMA 2.

$$
F_{l}=\frac{1}{x_{1}^{l}}\left((-1)^{l} \frac{x_{2}^{l}}{l((l-2) !)}+\sum_{k=1}^{l-2}(-1)^{l-k} \frac{x_{1}^{k} x_{2}^{l-k-1} x_{k+2}}{(l-k-1) !}-x_{1}^{l-1} x_{l+1}\right), \quad l=4,5, \ldots, n-2 .
$$

ТеОРема 3. Инварианты коприсоединенного представления, полученные для случая $\lambda=0$, функционально независимы. Функционально независимы и инварианты, полученные для случая $\lambda \neq 0$.

Автор выражает благодарность В.В.Трофимову за ценные обсуждения.

\section{СПИСОК ЛИТЕРАТУРЫ}

[1] Трофимов В. В. Введение в геометрию многообразий с симметриями. М.: Изд-во МГУ, 1989. 